\section{Der Junggebliebene}

Professor Michael Laniado leitet das Institut und Poliklinik für Radiologische Diagnostik des Dresdeners Universitätsklinikums Carl Gustav Carus. Auf dem diesjährigen Deutschen Röntgenkongress wurde ihm die Ehrenmitgliedschaft der DRG verliehen. Und das ist kein Zufall.

Das Besondere an unseren Ehrenmitgliedern ist, dass sie ein Faible haben. Normalerweise für die Radiologie oder Medizinphysik oder für eine Subspezialität der Radiologie - manchmal aber auch für ganz besondere andere Leidenschaften. Michael Laniado zum Beispiel liebt das Entertainment. Natürlich liebt er auch seinen Beruf. Und er ist zu Recht ein bisschen stolz auf das, was er geschafft hat. Und das, was er als Lehrstuhlinhaber und erfahrener Radiologe erreicht hat, gibt er mit Freuden weiter an die werdenden Radiologinnen und Radiologen. „Der Patient muss im Mittelpunkt stehen“, ist seine Devise. Und die Show? "Ja, das hat sich so ergeben, dass ich bei der Professorennacht, die in 16 deutschen Städten stattfindet, mitmachen durfte“, erklärt Laniado. „Da konnte ich mich regelrecht austoben und zeigen, dass ich zum einen ein bisschen was von Musik verstehe und zum anderen noch nicht total verkalkt bin!“ Die Professorennacht ist übrigens eine lange Partynacht für Studenten verschiedener deutscher Unistädte, bei der die eigenen Dozenten eingeladen werden, als DJ ihre Musik aufzulegen. Ja, Michael Laniado kann auch David Bowie, Michael Jackson und die Stones.

Und generell scheint er junggeblieben und unbeschwert - kein verstaubter Silberrücken, dem jetzt eben noch ein Preis verliehen wird. Und Laniados Liste ist nicht kurz - da reihen sich bereits mehrere Ehrenmitgliedschaften, etwa der polnischen oder der österreichischen Röntgengesellschaft, aneinander. Darunter ist auch der Kernspintomografiepreis 1997. Aber Laniado war schon immer bewusst, beschreibt er, dass es der Zufall ist, der das Leben bestimmt.

Nach dem Abitur weiß er zuerst nicht, welche Richtung er einschlagen soll. Ist es ratsam, den Arztweg einzuschlagen und wie der Vater, ein Chirurg, oder der Bruder, auch Mediziner, diese Laufbahn zu wählen? Ein Vorbild war sein Vater allemal. „Er hat sich nie über die Fülle an Arbeit beklagt, und er war ein extrem pflichtbewusster Mensch.“ Eine entspannte Familie scheint das zu sein. Und dann: der Zufall. Bei einem Urlaub auf Korsika 1972 lernt Laniado Leute kennen, die ihn darin bestärken, die für ihn passende Entscheidung zu fällen und auf sein Bauchgefühl zu hören. Das tut er auch. Er ist passionierter Sportler - und denkt sich: Wenn schon Medizin, dann Sportmedizin. Also führt es den gebürtigen Düsseldorfer vom Rhein an die Spree. Er nimmt 1973 sein Studium an der Freien Universität Berlin auf. In der Physiologie lernt er Professor Kirsch kennen. Es wird eines seiner großen Vorbilder. Er lässt sich von ihm für die Herz-/Kreislaufphysiologie begeistern. Gern würde Kirsch Laniado schließlich habilitieren lassen, aber - und hier hilft kein Zufall - „Es gab einfach zu viele habilitierte Physiologen zu der Zeit“, sagt Laniado, „also sammelte ich erst mal experimentelle Erfahrung." So verschlägt es ihn in die Radiologie am Klinikum Charlottenburg und zu Schering im Berliner Wedding. Den Hinweis, es dort zu probieren, gab ihm Professor Kirsch im Jahr 1983. Eine gute Entscheidung: Laniado arbeitet bei Professor Felix in einem BMBF-geförderten Forschungsprojekt zu den neuen MR-Kontrastmitteln. Derweil lernt er Professor Claussen kennen, Oberarzt bei Professor Felix - in der Radiologie. „So ergab sich auf einmal eine neue Möglichkeit. „Claussen und ich wurden Freunde, und so fand ich meinen Weg in die Radiologie. Bis 1988 war ich in Berlin, bis Claussen seinen Ruf nach Tübingen erhielt, und der Zufall wollte es so, dass er mehrere Kollegen mitnahm - ich gehörte dazu. “ In Tübingen hält es Laniado zwölf Jahre. Dann kommt er, der Ruf - nach Dresden, an die Technische Universität, wo er noch heute den Lehrstuhl innehat.

Was hat ihn eigentlich in der Berliner Zeit geprägt? „Außer der szenigen Atmosphäre und einzigartigen Kulturlandschaft?" schmunzelt er, „Das wissenschaftliche Arbeiten. Meine Doktorarbeit habe ich in der Kinderklinik bei Frau Prof. Ballowitz

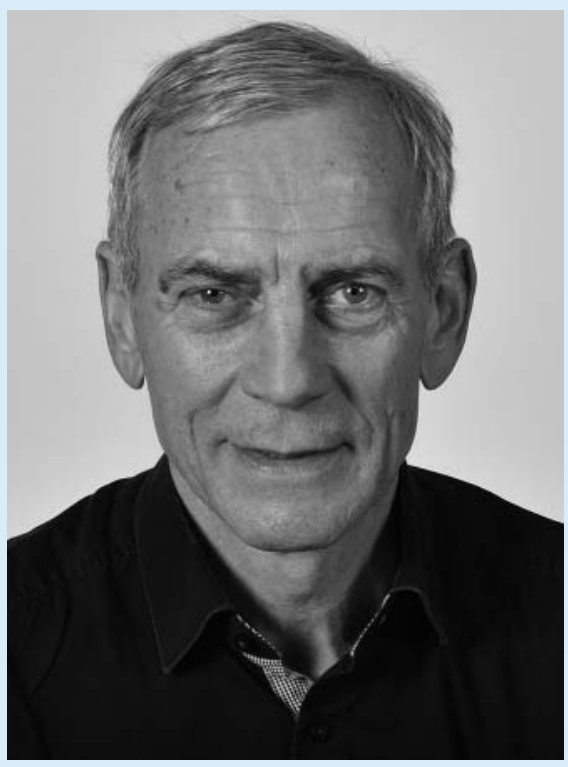

Prof. Dr. Michael Laniado, Dresden

geschrieben, sie war eine phantastische Betreuerin. Das war der Lackmustest. Das erste, was ich vor Beginn der Doktorarbeit gefragt wurde, war, ob ich gut im deutschen Aufsatz bin. “ Der Autor von insgesamt 140 wissenschaftlichen Arbeiten hat sicherlich jegliche Sorge vor dem Schreiben längst abgelegt. „Die akademische Radiologie macht mir schon immer Freude, und auch, wenn sie Stress und Verzicht bedeutet, so ist das Gegengewicht hochgradig positiv. Zum Beispiel gibt mir der Patientenkontakt sehr viel. Und er ist so wichtig, denn Radiologie muss ein Gesicht haben. Deshalb: möglichst viel davon!“

Man merkt Laniado an, dass er Freude an seinem Job hat. „Als Uni-Professor im Zentrum der Medizin zu stehen und Ansprechpartner für Kollegen sein, gerade im interdisziplinären Umfeld - ja, Spannungsfeld - das ist ein Hochgefühl." Worin liegt denn dabei die größte Herausforderung? „Einem Traumatologen muss ich auf einer Aufnahme keinen gebrochenen Knochen erklären, sondern ich muss wesentliche Informationen geben, wichtige Dinge beisteuern und dabei die zentrale Rolle des Radiologen stärken, das heißt: in diesem Spannungsfeld überhaupt bestehen! Und dabei stellen wir als Radiologen die Weichen, etwa in einer Tumorkonferenz. Ich 
sehe uns auf jeden Fall als letzte echte Allgemeinmediziner, aber auch als Spezialisten.“

Michael Laniado ist wichtig, diese Einstellung an die jungen Kollegen weiterzugeben. Vor allem dies: „Kommuniziert gut! Versetzt euch in die Rolle des Zuweisers, der einen gut strukturierten Befund braucht. Ich sehe es ähnlich wie Michael Forsting, Lehrstuhlinhaber in Essen: Ein kurzer Befund ist ein guter Befund!“ Und er weiß: „Jeder macht Fehler, deshalb ist Sorgfalt so wichtig. Die Vorgeschichte des
Patienten zu erfragen ist Teil dieser Sorgfalt und Präzision, die wir brauchen. Schließlich wird man als Radiologe ständig beobachtet, teilweise von unausgeschlafenen, übellaunigen Chirurgen - da muss man gewappnet sein“, erklärt er mit einem Lächeln. Er nimmt's halt nicht so schwer.

Und wenn dann die schlechtgelaunten Chirurgen befriedet und die DJ-Nacht geschafft sind, wie entspannt er dann? „So lang die Puste reicht, mache ich Sport. Fußball - nicht besonders gut, aber begeistert! Und zwar mit einer sehr netten Truppe von
Freunden. Als Jugendlicher vor allem Tennis, ich war sogar ganz gut. Aber Dresden, diese wunderschöne Stadt mit all ihren historischen Facetten, lenkt mich schon genug vom Stress ab.“

Vom Rhein, über die Spree bis zur Elbe. Der Weg war nicht immer eben. Aber Professor Michael Laniado wusste ja von Anfang an, dass der Zufall sowieso den Weg ganz plötzlich umleiten kann. Er hat ihn aber unter anderem zu einem Spezialisten in abdominaler Radiologie, und zwar auf europäischer Ebene, gemacht. Hut ab. 